\title{
Factores asociados a la calidad en el servicio en Centros de Acondicionamiento Físico a partir del modelo SERVQUAL
}

Factors associated with service quality in fitness centers based on the SERVQUAL model

\author{
Davinson Mosquera-González iD \\ Especialista en Analítica, Institución Universitaria Escolme, Medellín- Colombia, \\ davmosgon@gmail.com \\ Orfa Nidia Patiño-Toro \\ Administradora Tecnológica, Institución Universitaria Escolme, Medellín- Colombia, \\ cies4@escolme.edu.co \\ Daniel Mauricio Sánchez-Díez iD \\ Profesional en Mercadeo, Institución Universitaria Escolme, Medellín- Colombia, \\ dmsanchezd@escolme.edu.co \\ Juan Felipe Agudelo-Cardona iD \\ Profesional en Mercadeo, Institución Universitaria Escolme, Medellín- Colombia, \\ jfagudeloc@escolme.edu.co \\ Diana Marcela Ospina-Mazo \\ Profesional en Mercadeo, Institución Universitaria Escolme, Medellín- Colombia, \\ dmospinam@escolme.edu.co \\ Juan Felipe Bermúdez-Bedoya \\ Estudiante de Ingeniería de Sistemas, Universidad Nacional de Colombia, \\ Medellín-Colombia, jfbermudezbe@unal.edu.co
}

Cómo citar / How to cite

Mosquera-González, D., Patiño-Toro, O. N., Sánchez-Díez, D. M., Agudelo-Cardona, J. F., Ospina-Mazo, D. M., Bermúdez-Bedoya, J. F. (2019). Factores asociados a la calidad en el servicio en Centros de Acondicionamiento Físico a partir del modelo SERVQUAL. Revista CEA, 5(9), 13-32. https://doi.org/10.22430/24223182.1253

Recibido: 25 de septiembre de 2018

Aceptado: 26 de noviembre de 2018

\section{Resumen}

La presente investigación, con enfoque cuantitativo, tiene como objetivo medir los factores asociados a la calidad en el servicio en Centros de Acondicionamiento Físico (CAF), a partir del modelo 
Factores asociados a la calidad en el servicio en Centros de Acondicionamiento Físico a partir del modelo SERVQUAL

SERVQUAL. Para ello, se propone un modelo estructural, que contempla las 5 dimensiones del SERVQUAL, al que se le realizó un análisis para valorar su capacidad explicativa en este tipo de servicios. Se empleó una muestra de 279 usuarios de CAF; el instrumento de recolección consta de 20 ítems que posteriormente se agruparon en 5 constructos: confiabilidad; bienes tangibles/infraestructura; responsabilidad; servicio recibido (en términos de seguridad) y servicio esperado (en términos de empatía). Los resultados arrojaron que el modelo propuesto es significativo al momento de explicar el fenómeno estudiado, puesto que las 10 relaciones hipotéticas planteadas superan el umbral de significancia, siendo la asociación entre fiabilidad y elementos tangibles la de mayor grado, con un coeficiente $V$ de Cramer de 0.806 . Con lo anterior, se arroja evidencia a favor del uso de modelos basados en el SERVQUAL, para la medición de la calidad en tipos de servicios deportivos.

Palabras clave: modelo SERVQUAL, medición de servicios deportivos, medición de la calidad de servicios, Centros de Acondicionamiento Físico.

\begin{abstract}
The objective of this quantitative study is to measure the factors associated with service quality at fitness centers based on the SERVQUAL model. For that purpose, this work proposes a structural model that considers the 5 dimensions in the SERVQUAL model, which was analyzed to evaluate its explanatory power for such type of services. The sample was composed of 279 members of training centers. The collection instrument was a questionnaire that contained 20 items grouped into 5 constructs: reliability, tangible assets/infrastructure, responsibility, provided service (in terms of safety), and expected service (in terms of empathy). The results show that the proposed model can significantly explain the phenomenon under study because the 10 hypothetical relationships suggested in this article exceeded the significance threshold. In particular, the association between reliability and tangible assets reached the highest value, a Cramér's $V$ coefficient of 0.806 . This evidence supports the use of SERVQUAL-based models to measure the quality of sports services.
\end{abstract}

Keywords: SERVQUAL model, sports services measurement, service quality measurement, fitness center.

\title{
1. INTRODUCCIÓN
}

En la actualidad, se ha resaltado la importancia de la medición como un proceso que permite la generación de información con características de confiabilidad y generación de confianza por el conocimiento objetivo que se obtiene a través de esta (Maul, Mari, Torres \& Wilson, 2018). Es así como en el contexto empresarial actual, marcado por la alta competitividad, la medición de la calidad se convierte en algo ineludible, donde se presenta la aplicación de procesos constantes de análisis y valoración de la calidad de los servicios, cuyos datos se convierten en factores determinantes en la mejora continua (Hemalatha, Dumpala \& Balakrishna, 2018).

Lo anterior, entendiendo la complejidad del contexto actual, en el que el segmento de servicios ha presentado un crecimiento significativo en los diferentes países del mundo, especialmente influenciados por variables asociadas con el aumento de ingresos económicos, expansión de la población en las ciudades y transformaciones en los parámetros de conducta (Cuadrado, 2016). En 
tal sentido, adquieren importancia la medición, modelos y herramientas que permitan determinar la percepción de los usuarios asociada con la calidad de los servicios ofrecidos por las distintas organizaciones, favoreciendo con esto el emprendimiento de acciones de mejora en las áreas que presenten deficiencias (Murillo \& Saurina, 2013).

De la misma manera, se expone el modelo Servqual como mecanismo de medición para hallar las posibles brechas entre la calidad entregada por las organizaciones y las perspectivas que los clientes tienen sobre la calidad de los servicios, lo que representa una valiosa oportunidad para la toma de decisiones coherentes con la realidad, necesidades y satisfacción de los usuarios (Chhabra, 2013).

Análogamente, en la segunda parte del texto se relacionan aspectos inherentes a la importancia de la medición de la calidad en el sector deportivo, y se describen algunos de los instrumentos más utilizados en procesos de evaluación de la calidad percibida por los espectadores, participantes o asistentes en diferentes ámbitos del deporte. Dentro de este marco, se aprecia la relevancia de conocer e identificar las diversas dimensiones del servicio para tener la capacidad de generar estrategias permanentes de mejora y poder alcanzar resultados efectivos: desde el entendimiento de necesidades, perspectivas y resultados esperados por los usuarios, mediante la aplicación de instrumentos de medición pertinentes para la correcta gestión de la calidad institucional (Morquecho-Sánchez, Medina-Rodríguez, Morales-Sánchez, Medina \& Duelos-Martínez, 2013).

La revisión de información en el contexto de entidades en el campo deportivo, se desarrolla con el objeto identificar los aportes de expertos; además de esto, permite evidenciar semejanzas y controversias de los estudios encontrados, en relación con técnicas de análisis, supuestos, entre otros; de tal forma que, se contribuya desde lo metodológico a nuevas investigaciones, así como en actividades de formación y apropiación de conocimientos ligados con la estimación de la calidad de los servicios.

Es así como esta investigación tiene como objetivo principal proponer un modelo estructural basado en el SERVQUAL, para la medición de los factores asociados a la calidad del servicio en Centros de Acondicionamiento Físico (CAF). A su vez, se busca determinar el grado de asociación entre las dimensiones contempladas en mencionado modelo. Con esto, se brindarían aportes útiles en favor de la realización de procesos de evaluación constantes de la calidad en diferentes escenarios o espacios organizativos.

\section{MARCO TEÓRICO}

El entorno complejo, dinámico e incierto de hoy, convierte a la calidad de los servicios en factor crítico y diferencial para el logro de la ventaja competitiva en el mercado (Manulik, Rosińczuk \& Karniej, 2016), en el que se ha presentado un drástico cambio de escenarios para el desarrollo de las actividades. Por tal motivo, su medición y vigilancia permanente se traducen en asuntos prioritarios para las empresas (Gulc, 2016). En efecto, se reconoce que todas las actividades vinculadas con la calidad se deben alinear con el interés por alcanzar la ventaja competitiva en el mercado y con las metas institucionales (Panigrahi, Azizan \& Ali Khan, 2018). 
Factores asociados a la calidad en el servicio en Centros de Acondicionamiento Físico a partir del modelo SERVQUAL

La medición de la calidad del servicio adquiere trascendencia como consecuencia del análisis de la eficacia, dado que es la base para iniciar las estrategias de mejora e incorporación de prácticas dirigidas a potenciar los estándares de calidad y consecución de las metas trazadas por las organizaciones. No obstante, es importante la selección apropiada de los métodos a utilizar en el procedimiento, puesto que de lo contrario podrían presentarse inconsistencias y limitaciones en la integridad de las derivaciones de la evaluación (Gulc, 2016).

Por otra parte, el servicio es conceptualizado por Duque \& Pinzón (2012) como todas las acciones que se requieren para producir satisfacción en los clientes. Es por ello que la prestación de servicios involucra un cúmulo de actividades que deben integrarse de forma adecuada con el objeto de brindar a los usuarios siempre la mejor calidad posible (García, 2016). De igual forma, es importante resaltar el campo de servicios como la tercera rama de la economía, presentando un avance significativo, especialmente en los estados desarrollados. Este es un sector marcado por la diversidad de instituciones que convergen en él, y a través del cual se pretende lograr la transformación de los individuos, procesos y organizaciones (Vargas \& Aldana, 2015).

A su vez, conforme a los postulados de Takenaka, Nishino \& Nishikori (2018), todas las actividades implicadas en el servicio forman parte de la existencia humana, por ende, se aprecia que es conveniente manejarlas como sistemas que exponen alta complejidad, puesto que parten de la interacción de las personas. Análogamente, los servicios deben valorarse analizándolos desde distintos ángulos (satisfacción de empleados, usuarios, niveles de beneficios financieros y con relación a la valoración social.

\section{Medición de la calidad}

El concepto de calidad es definido por Goetsch \& Davis (2013) como un término dinámico relacionado con diferentes áreas o elementos: personas, productos, entornos, servicios, entre otros, que se orientan a la satisfacción de las expectativas, intereses o diversas necesidades de los usuarios. Por su parte Vargas \& Aldana (2015) afirman que la calidad es la base del desarrollo de las instituciones y factor esencial de los productos y servicios que estas proveen a los clientes.

En el contexto empresarial moderno, marcado por la alta competitividad, se convierte en algo ineludible la aplicación de procesos constantes de análisis y valoración de la calidad de los servicios que son entregados por parte de las instituciones a los distintos usuarios, como factores determinantes en su mejora continua (Hemalatha et al., 2018). Esto se hace cada vez más evidente, puesto que los usuarios están más informados y son más exigentes, por lo que los conocimientos de los niveles de satisfacción se convierten en instrumento que provee elementos importantes en la identificación de inconsistencias o deficiencias para tomar las acciones correctivas (Midor \& Kučera, 2018).

Es así que, en estos procesos de estimación de la calidad (atributos y requerimientos técnicos), se utilizan métodos para apreciar la calidad con la que se prestan los servicios, favoreciendo la detección de las áreas cuya mejora debe contribuir a la excelencia (Hemalatha et al., 2018). 
Es necesario considerar la importancia de la medición como un proceso que permite la generación de información con características de confiabilidad y generadora de confianza por el conocimiento objetivo que se obtiene a través de esta (Maul et al., 2018).

\section{Modelos de medición de la calidad}

Dentro de este contexto, los modelos de calidad son aquellos que facilitan la obtención de información necesaria para evaluar las condiciones en las que se prestan los servicios a los usuarios, facilitando la identificación de fortalezas e inconsistencias para el diseño e implementación estratégica de mejoras. En tal sentido, se han desarrollado diferentes instrumentos psicométricos robustos y rigurosos para examinar las condiciones reales de la calidad de los servicios y la percepción de los usuarios. Entre los modelos de evaluación más reconocidos se encuentran: «el modelo SERVQUAL, el modelo de implementación de funciones de calidad (QFD), el modelo Kano, el índice de mejora ( 1 i ) y el modelo de satisfacción de importancia (modelo IS)» (Ou, Mak \& Pan, 2017, p. 2). En el ámbito de la medición de la calidad en servicios y de acuerdo con la revisión realizada, se tiene que dos de los modelos más utilizados para el estudio de la perspectiva personal son: el modelo SERVQUAL, propuesto por Parasuraman, Zeithaml \& Berry (1988), que se detallará a continuación, y el modelo SERVPERF propuesto por Cronin \& Taylor (1992) que se enfoca específicamente en valorar la calidad del servicio por medio de la experiencia, bajo el supuesto de entender la calidad como parte intrínseca de la superioridad del servicio brindado. También es común el uso de metodologías de investigación de incidentes, en la que se supone que los clientes miden la calidad conforme a su propia experiencia, y en los que se evalúa la calidad a partir de las quejas interpuestas por los clientes (Gulc, 2016).

\section{Modelo SERVQUAL}

Para los autores Parasuraman, Zeithaml \& Berry (1988), quienes patentaron el modelo SERVQUAL (SERVice QUALity), la calidad hay que entenderla como un proceso integrado cuando se hace referencia al ámbito de los servicios. Este proceso inicia con las percepciones obtenidas vía estudios de mercado y que los directivos se forman respecto a las expectativas de los clientes, las cuales a su vez se construyen con la comunicación con otros usuarios del servicio tomando como base sus necesidades y experiencias individuales, además de lo que la propia empresa les transmita (Tabla 1) (Fragoso \& Espinoza, 2017).

El método SERVQUAL es una práctica que se ha extendido para evaluar la calidad deseada en la prestación de los servicios frente a la calidad real experimentada por los clientes al recibirlos (Manulik et al., 2016).

EI SERVQUAL es un modelo constituido por 22 variables, a partir de las cuales se evalúan las expectativas (lo que se espera), frente a la calidad del servicio recibido por los usuarios, asignando calificaciones y estableciendo las posibles diferencias entre estas; siendo una escala que permite obtener resultados con altos índices de confiabilidad y validez (Duque \& Pinzón, 2012), buscando el desarrollo de mejoras, que permitan potenciar la relación con el público y la atracción de clientes nuevos interesados. 
Factores asociados a la calidad en el servicio en Centros de Acondicionamiento Físico a partir del modelo SERVQUAL

Tabla 1. Dimensiones del modelo SERVQUAL y su descripción

Table 1. Dimensions and description of the SERVQUAL model

\begin{tabular}{l|l}
\hline \multicolumn{1}{c|}{ Criterio } & \multicolumn{1}{c}{ Descripción } \\
\hline Elementos tangibles & $\begin{array}{l}\text { Mide la apariencia de las instalaciones físicas, de } \\
\text { los equipos y la apariencia de los empleados }\end{array}$ \\
\hline Fiabilidad & $\begin{array}{l}\text { Mide la capacidad de la organización para cumplir } \\
\text { con lo prometido y hacerlo sin errores }\end{array}$ \\
\hline Capacidad de respuesta & $\begin{array}{l}\text { Mide la voluntad de ayuda a los usuarios y la } \\
\text { rapidez y la agilidad del servicio }\end{array}$ \\
\hline Seguridad & $\begin{array}{l}\text { Mide el conocimiento y la cortesía de los } \\
\text { empleados y su capacidad para inspirar confianza } \\
\text { y seguridad }\end{array}$ \\
\hline Empatía & $\begin{array}{l}\text { Mide la atención esmerada e individualizada, la } \\
\text { facilidad de acceso a la información, la capacidad } \\
\text { de escuchar y entender las necesidades }\end{array}$ \\
\hline
\end{tabular}

Fuente: (Fragoso \& Espinoza, 2017).

\section{Importancia de la medición de la calidad en el sector deportivo (CAF)}

Es evidente la expansión de las empresas de servicios, en las que gran cantidad de ellas presentan un alto índice de rotación de usuarios, especialmente en el plano deportivo, causando así una permanente preocupación en los propietarios. Lo anterior, propicia la búsqueda de estrategias para conservar y fidelizar los actuales, así como atraer nuevos usuarios. De tal forma que se desarrolla un creciente interés por atender los principales factores que condicionan la permanencia de los beneficiarios del servicio, conforme a su percepción (calidad, valor, satisfacción, entre otros) (Bernal, 2014).

Considerando que en la actualidad no es exclusivamente el monto monetario el determinante de las decisiones para la utilización de algún servicio, sino que existen componentes (satisfacción, la percepción de la calidad y el valor) que otorgan mayor ventaja competitiva a las instituciones, a partir de mecanismos enfocados en la fidelización de los usuarios. Por ende, es clave la identificación de instrumentos apropiados para la medición de la calidad, puesto que contribuye a que los responsables de los espacios deportivos examinen las condiciones, potencialidades y limitaciones para la introducción de mejoras a que haya lugar en aras de la maximización de la calidad y fidelización de los beneficiarios; atendiendo a aspectos en cuanto a calidad de la infraestructura, equipos, entorno, sistematización, atención al usuario, costos, comunicación, imagen de la entidad, etc. (Ramírez, Lorenzo \& López, 2017).

En todo caso, la transformación de los enfoques del mercado, inicialmente orientado al producto y pasar a centrarse en los clientes (expectativas, deseos, necesidades), implica cambios significativos en la forma de llegar al público; a través de la prestación de servicios en la parte deportiva. Adicional a esto, en un escenario de mayor competencia (masificación de la oferta de gimnasios y centros de acondicionamiento físico) inciden en la adopción de herramientas precisas de medición, aportando a la excelencia en la gestión de calidad (García, Bernal, Lara \& Galán, 2013).

De igual forma, otros autores han expuesto diferentes herramientas con los que se ha buscado evaluar la calidad percibida por los clientes en entidades dedicadas a entregar servicios en el ámbito 
del deporte, entre los cuales se pueden mencionar a Theodorakis, Kambitsis \& Laios (2001), quienes por medio del instrumento SPORTSERV analizan la calidad y la satisfacción de los espectadores con respecto a variables tales como: accesibilidad, confiabilidad, capacidad para ofrecer respuestas adecuadas y oportunas, equipos y materiales y aquellas asociadas a la seguridad de los usuarios que asisten a eventos deportivos.

Por otra parte, a través de la herramienta de medición ICPAF se han evaluado elementos vinculados con la calidad de los programas deportivos ofrecidos por instituciones de este sector, como es el caso de: el instructor, infraestructura física, las actividades ofrecidas, las competencias del personal y calidad de la información suministrada (Morales, Hernández-Mendo \& Blanco, 2005). Dentro de este marco, también se ha considerado el mecanismo HAFSQ propuesto por Dhurup, Singh \& Surujlal (2006), mediante una serie de 39 variables se miden ocho dimensiones de la calidad del servicio ofrecido, donde se incluyen: personal, sistematización y grupo de especialistas, utilidad y distribución de información, el entorno y facilidad de acceso, diseño y funcionalidad, atractivo de la infraestructura de la entidad, entre otros.

Mientras tanto, Rial, Varela, Rial \& Ral (2010), a partir del método QSport-10 examinaron aspectos, en especial la infraestructura y el personal responsable, como factores claves de la percepción acerca de la calidad del servicio en instituciones relacionadas con el deporte. También, se puede mencionar la herramienta EVENTQUAL, indagada por Calabuig, Burillo, Crespo, Mundina \& Gallardo (2010), con la que se examina la percepción sobre calidad y valoración de los asistentes a eventos de atletismo, tomando como factores: la infraestructura (instalaciones, equipos, materiales), el personal, aquellos servicios adicionales y el acceso; buscando identificar elementos críticos en la satisfacción y fidelización del público.

De igual modo, por medio del modelo CECASDEP se mide la percepción y satisfacción de los usuarios de los programas deportivos municipales, y se toman como puntos de referencia: instalaciones, espacios, camerinos, programas propuestos para la actividad física y los entrenadores (Gálvez \& Morales, 2011). Adicionalmente, se ha empleado la denominada escala CALIDFIT para evaluar el nivel de satisfacción de los usuarios y el nexo con la percepción de la calidad en centros de acondicionamiento físico; en esta, se toman en consideración componentes importantes como: personal, infraestructura, entorno, cantidad de equipos y los servicios (García, Cepeda \& Martín, 2012).

A continuación, se presenta el modelo propuesto para este estudio con las respectivas relaciones hipotéticas a validar: 


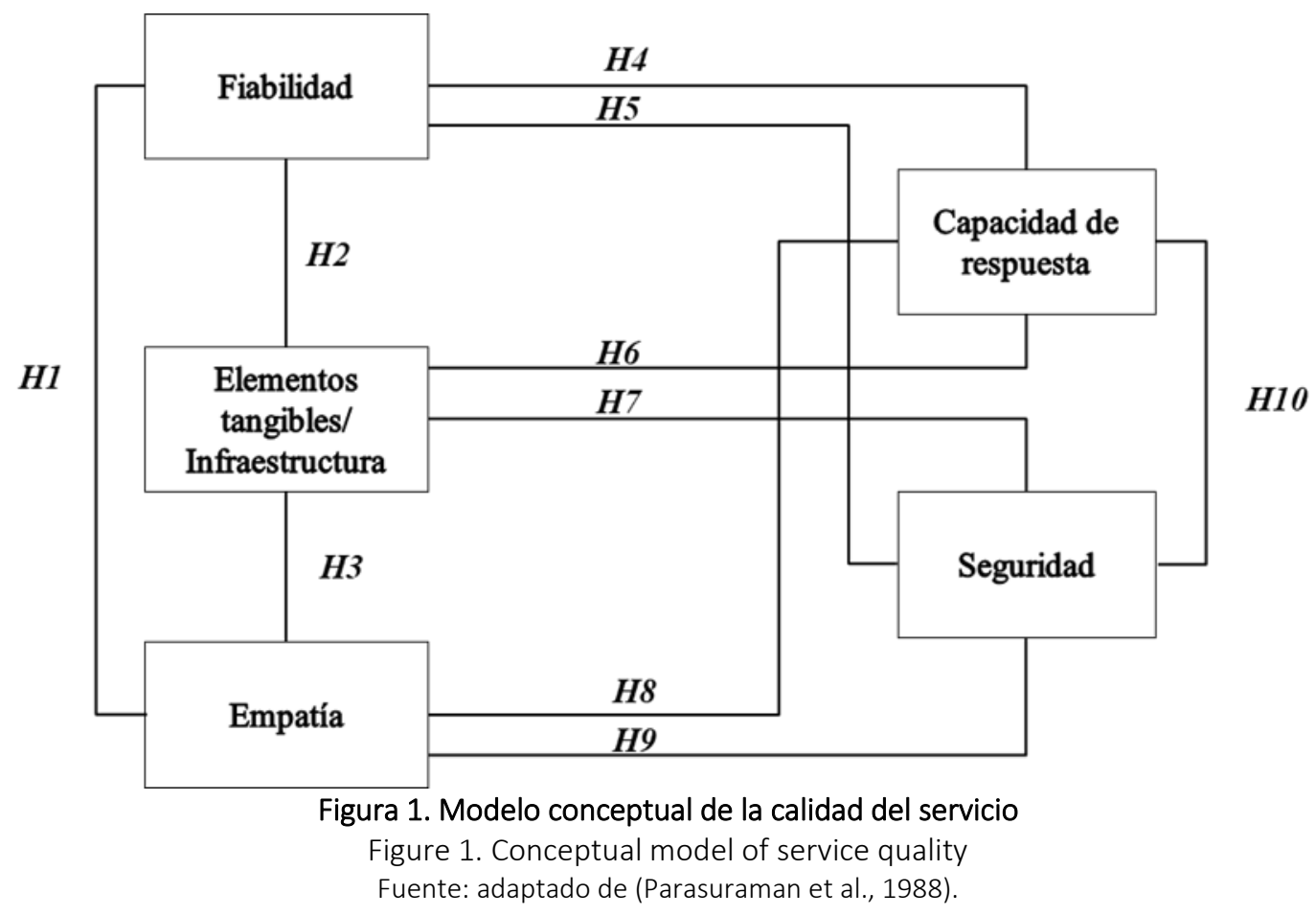

\section{METOdOLOGÍA}

\section{Muestra}

Para el desarrollo de la presente investigación se utilizó una muestra compuesta por 279 usuarios, hombres y mujeres, de 3 Centros de Acondicionamiento Físico de la ciudad de Medellín. El procedimiento de muestreo fue no probabilístico a conveniencia. La delimitación de la población se basó en que los participantes tuvieran entre 18 y 50 años de edad y que pertenecieran a los estratos socioeconómicos 3, 4, 5 y 6; cuyos porcentajes de participación fueron de 46 \%, 34 \%, 19 \% y 1 \%, respectivamente. Se recolectaron 93 encuestas en cada centro de acondicionamiento físico. Se tuvo que el $51 \%$ de los participantes eran hombres y el $49 \%$ mujeres; quiénes tenían nivel de formación técnico, tecnólogo, profesional y especialización; en una proporción de 23 \%, 32 \%, 35 \% y 10 \%; correspondientemente. Entre los criterios de exclusión se tenía no ser usuario frecuente de los centros de acondicionamiento, es decir, no haber adquirido ningún plan o paquete de servicio y hacer uso de dos o más de los centros de acondicionamiento, de este estudio, al mismo tiempo.

\section{Procedimiento}

Inicialmente se elabora un instrumento de recolección de datos, de acuerdo con la revisión de literatura realizada, el cual tiene como propósito recolectar información sobre la percepción que expresan los usuarios de los Centros de Acondicionamiento Físico, respecto a las cinco dimensiones contempladas por el modelo SERVQUAL; elementos tangibles, fiabilidad, capacidad de respuesta, seguridad y empatía (Parasuraman et al., 1988). El cuestionario está conformado por 20 ítems, adaptados a partir de los 22 contemplados por el modelo Servqual. Tal modificación está sustentada 
en el hecho de que las relaciones hipotéticas planteadas en el modelo estructural de la Figura 1, varían respecto a las del modelo Servqual. Dado esto, se conforman los 5 constructos que contienen 2 o más variables cada uno; la fiabilidad, cuatro ítems; la capacidad de respuesta, tres ítems; tanto la dimensión de seguridad como la de empatía, con cinco ítems; y, por último, la dimensión de elementos tangibles con 3 elementos. Para el llenado de los datos se emplea una escala Likert de cinco puntos, en el que la primera opción de respuesta (de izquierda a derecha), es decir, el número 5 implica que los usuarios están completamente de acuerdo con la afirmación, y 1 en total desacuerdo con lo expuesto en el ítem.

La investigación se enmarca en un estudio de alcance descriptivo, dado que el objetivo de la misma es desarrollar una imagen o fiel representación (descripción) del fenómeno estudiado, a partir de sus características; puntualmente, se miden variables o conceptos, con el fin de especificar las propiedades importantes de comunidades, personas, grupos o fenómeno bajo análisis. El énfasis está en el estudio independiente de cada característica, es posible que de alguna manera se integren las mediciones de dos o más características con el fin de determinar cómo es o cómo se manifiesta el fenómeno y de esta manera obtener una estructura base del modelo teórico a presentar (Grajales, 2000).

Es de mencionar, que los encuestados participaron de forma voluntaria y anónima. Adicionalmente, se realizó una prueba piloto con 30 usuarios, con el fin de corroborar la claridad y entendimiento de las preguntas.

\section{Análisis de los datos}

Dado que el objetivo principal de la presente investigación ha sido la aplicación y validación de un modelo de calidad del servicio, el primer paso consistió en la selección de las variables a estudiar del modelo, las cuales están concentradas en los constructos confiabilidad, bienes tangibles/infraestructura, responsabilidad, servicio recibido (en términos de seguridad) y servicio esperado (en términos de empatía). Para ello se diseñaron las variables de acuerdo con la información útil a extraer, para el objetivo de la investigación y se seleccionaron todas aquellas que, según se explica en el modelo teórico, se definen como predictores o relacionados con la percepción del servicio y las expectativas o calidad esperada.

Las escalas de medida utilizadas, junto con cada uno de los constructos y el instrumento en general, se comprobó a través del método estadístico análisis factorial confirmatorio, el cual fue desarrollado utilizando el software estadístico SPSS versión 25. Inicialmente se realizó un análisis de la validez convergente de los constructos, en el que se calcularon las cargas factoriales estandarizadas para el posterior cálculo de los promedios de cada constructo. En lo referente a la correlación entre variables, se procedió a calcular el test de esfericidad de Barlett y la medida de adecuación de la muestra KaiserMeyer-Olkin (KMO) (Kaiser, 1970).

Posteriormente, se realizó un análisis de la validez discriminante, el cual se llevó a cabo mediante la comprobación de que el intervalo de confianza en la estimación de la correlación entre cada par de factores no contuviera el valor uno (Anderson \& Gerbing, 1988). 
Luego se procedió a identificar la fiabilidad de la escala de medida y verificar la capacidad explicativa del modelo SERVQUAL, para ello se calculó el alfa de Cronbach para las respectivas escalas de cada constructo. Posteriormente, se realizaron las pruebas de hipótesis en las que se estimaron estadísticos para el modelo estructural propuesto. En este punto, el interés se centró en determinar el grado de asociación entre constructos, cuyas relaciones están expresadas en el modelo de la Figura 1. Para ello se utilizó el estadístico $\vee$ de Cramer, propuesto por Cramer (1946), el cual es un coeficiente basado en el estadístico chi cuadrado, que permite determinar nivel de asociación entre variables. Sus valores oscilan entre 0 y 1 ; siendo números cercanos a 1 los de mayor grado de asociación y valores cercanos a 0 , los de menor. La justificación del uso de la $\mathrm{V}$ de Cramer en esta investigación radica en que este estadístico corrige el problema de las diferencias entre tamaños de las diferentes formas que podían tomar las variables, es decir, no afecta el tipo de la tabla de contingencia utilizada.

\section{RESULTADOS}

\section{Validez convergente}

En primera instancia se tiene la validez convergente del modelo, la cual evalúa el grado en que la medida de los ítems que recogen un mismo concepto están correlacionados, donde se analiza la fiabilidad en dos niveles: fiabilidad de los ítems observables y la fiabilidad de los constructos (Calvo, Martínez \& Juanatey, 2013). En la validación de este estudio no fue necesario eliminar ningún indicador, dado que su carga factorial estandarizada arrojó resultados superiores a 0.6, lo que es considerado como evidencia de que el modelo es fiable (Bagozzi \& Yi, 1988) y el promedio de las cargas fue superior a 0.7 para todos los constructos (Hair, Anderson, Tatham \& Black, 2001), tal como se muestra en la Tabla 2.

En lo referente a la correlación entre variables, inicialmente se realizó el test estadístico de esfericidad de Barlett, el cual detecta la presencia de correlación entre variables, su valor p debe ser inferior a 0.05 o 0.01. Por otra parte, el valor de la medida de adecuación de muestreo de KaiserMeyer-Olkin (KMO), cuyos valores comprenden entre 0 y 1.

Se observa en la Tabla 3 que los coeficientes arrojados por el software SPSS para cada uno de los constructos alcanza a cumplir con los criterios de aceptación de Lévy, Martín \& Román (2006), en el que valores KMO alrededor de 0.7 o superior, implican factibilidad para realizar la técnica de reducción de datos.

\section{Validez discriminante}

El análisis de validez discriminante se llevó a cabo mediante la comprobación de que el intervalo de confianza en la estimación de la correlación entre cada par de factores no contuviera el valor uno (Anderson \& Gerbing, 1988). La Tabla 4 evidencia que todos los casos cumplen con dicho criterio y de esta forma se constata que, la validez del instrumento utilizado mide en alto grado lo que se pretendía medir. 
Tabla 2. Validez convergente de cargas factoriales estandarizadas Table 2. Convergent validity of standardized factor loadings

\begin{tabular}{|c|c|c|c|}
\hline Constructo & Ítem & Cargas factoriales estandarizadas & $\begin{array}{c}\text { Promedio de cargas } \\
\text { factoriales estandarizadas }\end{array}$ \\
\hline \multirow{3}{*}{ Capacidad de } & CR1 & 0.733 & \multirow{3}{*}{0.782} \\
\hline & CR2 & 0.749 & \\
\hline & CR3 & 0.862 & \\
\hline \multirow{5}{*}{ Empatía } & E1 & 0.859 & \multirow{5}{*}{0.765} \\
\hline & E2 & 0.840 & \\
\hline & E3 & 0.681 & \\
\hline & E4 & 0.696 & \\
\hline & E5 & 0.748 & \\
\hline \multirow{3}{*}{ Elementos tangibles } & ET1 & 0.827 & \multirow{3}{*}{0.833} \\
\hline & ET2 & 0.876 & \\
\hline & ET3 & 0.796 & \\
\hline \multirow{4}{*}{ Fiabilidad } & F1 & 0.701 & \multirow{4}{*}{0.706} \\
\hline & F2 & 0.593 & \\
\hline & F3 & 0.769 & \\
\hline & F4 & 0.760 & \\
\hline \multirow{5}{*}{ Seguridad } & S1 & 0.806 & \multirow{5}{*}{0.734} \\
\hline & S2 & 0.687 & \\
\hline & S3 & 0.815 & \\
\hline & S4 & 0.732 & \\
\hline & S5 & 0.631 & \\
\hline
\end{tabular}

Fuente: elaboración propia con apoyo del software estadístico SPSS.

Tabla 3. Validación convergente del KMO y prueba de esfericidad de Bartlett Table 3. Convergent validation of the KMO and Bartlett's sphericity test

\begin{tabular}{lccc}
\hline Factor & Valor KMO & Valor Bartlett & $\begin{array}{c}\text { Cumple } \\
\text { criterios }\end{array}$ \\
\hline Capacidad de respuesta & 0.604 & 0.000 & Sí \\
\hline Empatía & 0.828 & 0.000 & Sí \\
\hline Elementos tangibles & 0.678 & 0.000 & Sí \\
\hline Fiabilidad & 0.705 & 0.000 & Sí \\
\hline Seguridad & 0.762 & 0.000 & Sí
\end{tabular}

Fuente: elaboración propia con apoyo del software estadístico SPSS. 
Factores asociados a la calidad en el servicio en Centros de Acondicionamiento Físico a partir del modelo SERVQUAL

Tabla 4. Intervalo de confianza de las variables del modelo

Table 4. Confidence interval of the variables in the model

\begin{tabular}{|c|c|c|c|c|c|}
\hline Constructos & $\begin{array}{c}\text { Capacidad de } \\
\text { respuesta }\end{array}$ & Empatía & $\begin{array}{c}\text { Elementos } \\
\text { tangibles }\end{array}$ & Fiabilidad & Seguridad \\
\hline $\begin{array}{l}\text { Capacidad de } \\
\text { respuesta }\end{array}$ & $\cdots$ & & & & \\
\hline Empatía & {$[0.205 ; 0.521]$} & $\ldots$ & & & \\
\hline Elementos tangibles & {$[0.191 ; 0.553]$} & {$[0.294 ; 0.680]$} & $\ldots$ & & \\
\hline Fiabilidad & {$[0.267 ; 0.639]$} & {$[0.217 ; 0.615]$} & {$[0.347 ; 0.684]$} & $\ldots$ & \\
\hline Seguridad & {$[0.258 ; 0.608]$} & {$[0.306 ; 0.696]$} & {$[0.321 ; 0.690]$} & {$[0.212 ; 0.578]$} & $\ldots$ \\
\hline
\end{tabular}

Posteriormente, se calculó el índice alfa de Cronbach, el cual es usado para medir la consistencia interna de una escala (Oviedo \& Campo-Arias, 2005); su valor oscila entre cero y uno, entendiendo que, entre más cerca se encuentre a uno, es mayor la consistencia interna de los ítems analizados (George \& Mallery, 2003); siendo así, el instrumento de medida utilizado presenta una adecuada fiabilidad, tal como se observa en la Tabla 5.

Tabla 5. Coeficiente de fiabilidad

Table 5. Reliability coefficient

\begin{tabular}{lc}
\hline Factor & Alfa de Cronbach \\
\hline Capacidad de respuesta & 0.822 \\
\hline Empatía & 0.871 \\
\hline Elementos tangibles & 0.867 \\
\hline Fiabilidad & 0.785 \\
\hline Seguridad & 0.843 \\
\hline
\end{tabular}

Fuente: elaboración propia con apoyo del software estadístico SPSS.

\section{Prueba de hipótesis}

Para esta etapa de la validación, se procedió a realizar la estimación del modelo estructural propuesto, para evaluar sobre qué base determinan la calidad los usuarios de los centros de acondicionamiento físico de Medellín, en donde se recogen las diversas hipótesis planteadas y se mide su grado de asociación por medio del estadístico V de Cramer; en la Tabla 6 se encuentran los valores obtenidos para el estadístico de asociación analizado y el modelo utilizado.

Los estadísticos considerados para esta fase de validación toman valores comprendidos entre cero y uno, en donde los valores próximos a la unidad, en valor absoluto, indican una fuerte relación entre dos variables y los valores próximos a cero indican que hay poca o ninguna relación entre los constructos (Abascal \& Grande, 2005). 
Tabla 6. Prueba de hipótesis - Grado de asociación de factores Table 6. Hypothesis testing - Degree of association between factors

\begin{tabular}{|c|c|c|}
\hline Hipótesis & Correlaciones entre variables & V de Cramer \\
\hline \multirow[t]{2}{*}{$\mathrm{H} 1$} & & 0.793 \\
\hline & Fiabilidad Empatía & \\
\hline $\mathrm{H} 2$ & Fiabilidad Elementos tangibles & 0.806 \\
\hline $\mathrm{H} 3$ & Elementos tangibles Empatía & 0.785 \\
\hline $\mathrm{H} 4$ & Fiabilidad Capacidad de respuesta & 0.783 \\
\hline H5 & Fiabilidad Seguridad & 0.781 \\
\hline $\mathrm{H} 6$ & Elementos tangibles Capacidad de respuesta & 0.778 \\
\hline $\mathrm{H} 7$ & Elementos tangibles Seguridad & 0.803 \\
\hline $\mathrm{H} 8$ & Empatía Capacidad de respuesta & 0.779 \\
\hline $\mathrm{H} 9$ & Empatía Seguridad & 0.794 \\
\hline $\mathrm{H} 10$ & Capacidad de respuesta Seguridad & 0.798 \\
\hline
\end{tabular}

De acuerdo con la teoría planteada se puede concluir que, los coeficientes de asociación calculados para las relaciones hipotéticas del modelo presentan valores positivos y significativos, indicando con esto una alta correlación entre las variables evaluadas en el análisis.

Por otro lado, los coeficientes $V$ de Cramer fueron extraídos del software SPSS y ubicados en una tabla de factores cruzados, que permitieran observar el grado de asociación entre las variables que hacían parte de las hipótesis y las que no lo hacían, con el fin, no solo de comprobar el grado de asociación para las relaciones hipotéticas, sino también corroborar cómo se comporta la asociación entre los demás constructos que forman parte del modelo. La Tabla 7 evidencia todas las relaciones establecidas entre las variables del modelo.

Tabla 7. V de Cramer

Table 7. Cramér's V

\begin{tabular}{|c|c|c|c|c|c|}
\hline & $\begin{array}{l}\text { Capacidad de } \\
\text { respuesta }\end{array}$ & Empatía & $\begin{array}{l}\text { Elementos } \\
\text { tangibles }\end{array}$ & Fiabilidad & Seguridad \\
\hline $\begin{array}{l}\text { Capacidad de } \\
\text { respuesta }\end{array}$ & 1.000 & & & & \\
\hline Empatía & 0.779 & 1.000 & & & \\
\hline $\begin{array}{l}\text { Elementos } \\
\text { tangibles }\end{array}$ & 0.778 & 0.785 & 1.000 & & \\
\hline Fiabilidad & 0.783 & 0.793 & 0.806 & 1.000 & \\
\hline Seguridad & 0.798 & 0.794 & 0.803 & 0.781 & 1.000 \\
\hline
\end{tabular}

Fuente: elaboración propia con apoyo del software estadístico SPSS. 
Factores asociados a la calidad en el servicio en Centros de Acondicionamiento Físico a partir del modelo SERVQUAL

Finalmente, se presenta en la Figura 2 el respectivo modelo estructural propuesto con los valores $\mathrm{V}$ de Cramer correspondientes, que representan el grado de asociación entre los constructos.

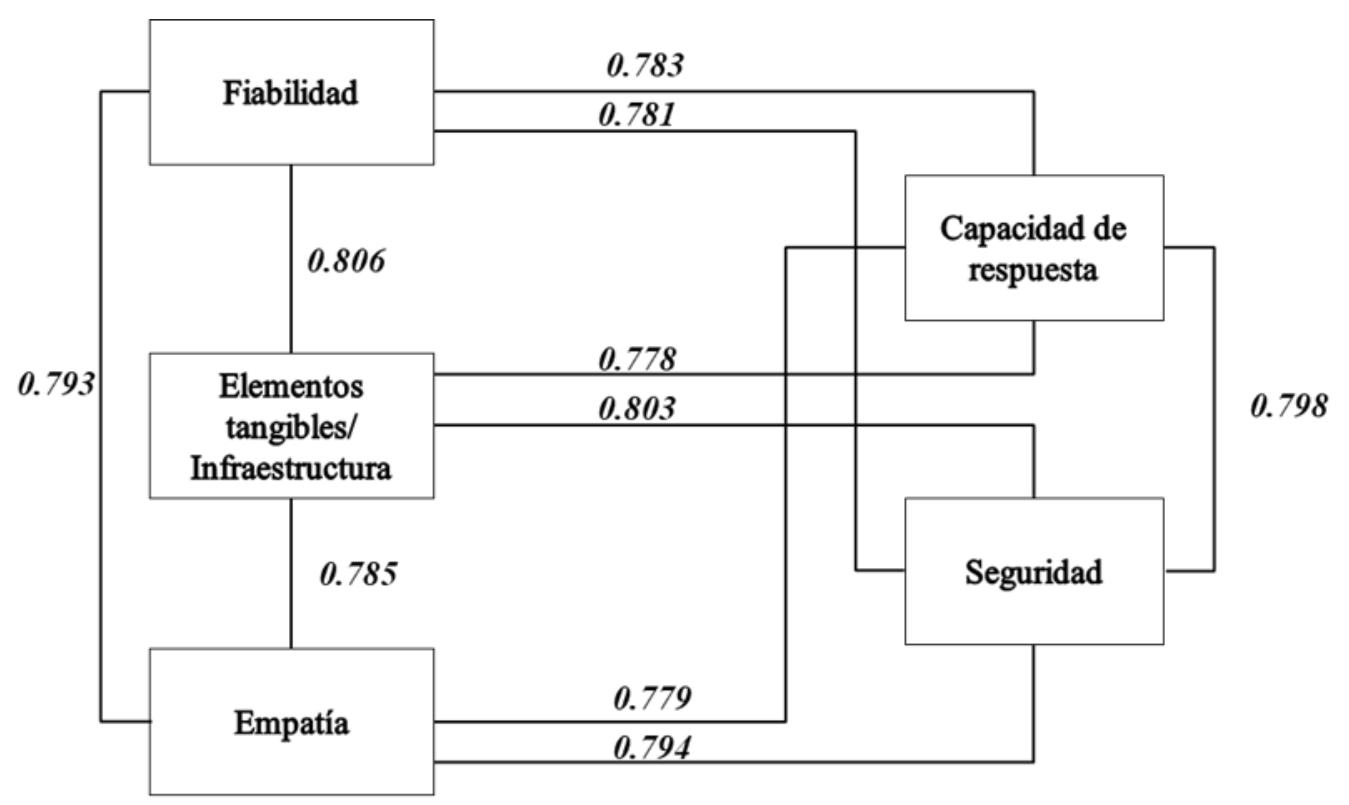

Figura 2. Modelo calidad del servicio con valores del coeficiente V de Cramer. Las flechas indican las hipótesis nulas que se validaron en la investigación.

Figure 2. Service quality model with values of Cramér's V coefficient. The arrows mark the null hypotheses validated in this work.

Fuente: elaboración propia.

\section{DISCUSIÓN}

Atendiendo al propósito de esta investigación, que buscó identificar la percepción de la calidad del servicio prestado por los principales Centros de Acondicionamiento Físico de Medellín, a partir del modelo Servqual, se encontró que todas las relaciones hipotéticas planteadas, son significativas. Para esta indagación se asumirá como significativo aquellos grados de asociación con coeficiente $V$ de Cramer igual o superior a 0.3, sustentados en Fierro (2010), quien afirma que en el marco de los estudios de las ciencias sociales se puede interpretar el coeficiente $\vee$ de Cramer de la siguiente manera: asociación baja $(0.0 \leq \mathrm{V}$ de Cramer $<0.2)$, asociación media $(0.2 \leq \mathrm{V}$ de Cramer $<0.3)$; $\mathrm{Y}$ asociación alta $(0.3 \leq \mathrm{V}$ de Cramer $\leq 1.0)$.

En el análisis, las variables dependientes fueron: servicio recibido respecto a la seguridad y servicio esperado respecto a la empatía. Las variables independientes fueron: fiabilidad, elementos tangibles/infraestructura y capacidad de respuesta.

Los resultados obtenidos de esas relaciones hipotéticas muestran que el servicio recibido en términos de la seguridad tiene una correlación significativa con la fiabilidad (0.806), dejando ver que la confianza transmitida por el personal técnico en el momento del entrenamiento físico puede generar una buena experiencia en el usuario y es la herramienta clave para cambiar o mejorar la percepción 
que este tiene del servicio prestado, ya que desde el nivel de acceso con el entrenador se pueden suplir las diferentes necesidades del cliente y lograr que él incremente su grado de satisfacción con el servicio recibido. De forma similar, la empatía tiene una fuerte asociación con la fiabilidad (0.793), para este grado de correlación se evidencia que un adecuado interés por parte del personal de apoyo para solucionar las dudas que tiene el deportista, presenta repercusiones positivas frente a la percepción de satisfacción de este.

Por otro lado, los elementos tangibles/infraestructura reflejan también un alto nivel de asociación con los constructos seguridad (0.803) y empatía (0.785), lo que puede estar indicando que las instalaciones físicas, equipos, personal y materiales de comunicación no solo permiten a los usuarios interactuar con el proveedor de servicios de entrenamiento personal y recibir atención personalizada, sino que también contribuye a la creación de un vínculo físico con la entidad, y de esta manera obtener beneficios a través del establecimiento de canales de comunicación que difundan información sobre líneas de servicio emergentes, sedes nuevas, mejores políticas de servicio y cualquier otro tipo de información que le de exclusividad a los usuarios antiguos y garantías a los que apenas estén ingresando.

Otra relación significativa que se presenta en el modelo es la que contrasta los constructos Empatía y capacidad de respuesta con el de seguridad, demostrando que un adecuado acompañamiento y seguimiento al usuario, ayuda a definir la percepción de los usuarios frente al beneficio que obtienen de las entidades de acondicionamiento físico y puede constituir el punto de referencia para determinar qué tan propenso está o no un usuario en aceptar o rechazar un servicio ofertado por otra entidad del sector de la salud física.

Finalmente, como complemento al modelo aplicado, se estableció la relación entre los factores capacidad de respuesta, elementos tangibles y fiabilidad, en donde se obtuvieron correlaciones muy significativas, lo cual refleja que, bajo condiciones de cumplimiento de expectativas y resolución de necesidades los usuarios tienden a estar más satisfechos y por ende a sentirse más motivados por seguir haciendo uso de los servicios les ofrezcan las empresas prestadoras de servicios de entrenamiento físico.

Ahora, autores como Martínez \& Martínez (2010) han cuestionado a nivel teórico y metodológico la idoneidad de algunos modelos multidimensionales de medición de calidad de servicio, entre ellos el modelo SERVQUAL y SERVPERF; así mismo Calabuig, F., Quintanilla, I, y Mundina, J. han realizado sus críticas en el sentido de la falta de especificad de estos modelos, sugiriendo que, en muchos de los casos de estudios sobre diferentes tipos de servicios, se requiera realizar adaptaciones. Pese a lo anterior, los resultados de esta investigación muestran que el modelo SERVQUAL presenta una significativa capacidad explicativa de un tipo de servicios deportivos (centros de acondicionamiento físico).

Por su parte, y en apoyo a los resultados de esta investigación, Morales et al. (2005) estudiaron la calidad del servicio en centros de entrenamiento físico, cuyos hallazgos plantearon, a través de análisis factorial confirmatorio, que el modelo SERVQUAL y adaptaciones del mismo son pertinentes e idóneas en ese tipo de servicios. Consecuentemente, Serrano \& Segado (2015) sostienen que, al realizar estudios sobre calidad de servicios en el ámbito deportivo o recreativo, los primeros 
Factores asociados a la calidad en el servicio en Centros de Acondicionamiento Físico a partir del modelo SERVQUAL

instrumentos a tener en cuenta son aquellos que utilizan o se basan el modelo SERVQUAL, puesto que el comportamiento en servicios deportivos no se sale del contexto de otros tipos de servicios.

Es así como el uso del modelo SERVQUAL en el ámbito de los servicios deportivos es tema de posiciones contrarias. Una posible explicación por la cual estudios similares arrojan posiciones contrarias, radica en el hecho de que variables como el país, ciudad, el nivel de reconocimiento de los centros de acondicionamiento físico e incluso la conformación de los constructos; es por esto que en algunos casos podría ser de utilidad efectuar inicialmente un análisis factorial exploratorio, con el fin de comprobar hasta qué punto estos instrumentos o los ítems que los conforman representan adecuadamente los constructos latentes de interés o diferentes dimensiones del mismo constructo (Mavrou, 2015), para posteriormente, sí realizar un estudio de carácter confirmatorio.

\section{CONCLUSIONES}

Los resultados de esta investigación arrojan evidencia explicativa en favor del uso del modelo SERVQUAL o adaptaciones del mismo en estudios sobre Centros de Acondicionamiento Físico y a su vez en servicios deportivos en general, puesto que todas las relaciones hipotéticas expresadas en el modelo propuesto resultaron ser significativas, según los respectivos coeficientes $V$ de Cramer. A partir de esto, se puede afirmar que el modelo planteado resulta de utilidad y referencia en el desarrollo de nuevos estudios, en el sentido que sirve de base para contemplar nuevas hipótesis o variaciones de las aquí expresadas. Lo anterior se hace pertinente en la idea de realizar estudios de este tipo, enmarcados en contextos particulares de ciudades, regiones o países, en los que se hace necesario la adaptación de constructos y variables sujetas de análisis.

Así mismo, el enfoque cuantitativo presentado en esta investigación, centró su interés en la validación y valoración de la capacidad explicativa de un modelo basado en el SERVQUAL. Los resultados arrojados dan pie a continuar estudios de enfoque cuantitativo, en los que se agregue una variable para medir la calidad de servicio global en los usuarios de CAF y determinar qué factores, de las dimensiones ya contempladas, presentan mayor influencia en esta nueva variable. Para ello será necesario la realización de un nuevo modelo estructural que represente las nuevas relaciones hipotéticas a considerar. Adicionalmente se propone continuar estudios de orden cualitativo, a modo complementario y de profundización sobre los hallazgos de esta investigación, contribuyendo así al entendimiento y conocimiento teórico en el análisis de percepción de calidad de servicio por parte de usuarios de diferentes actividades comerciales.

Además de las implicaciones teóricas de esta investigación, también se presentan una serie de impactos en el contexto productivo, en el que los resultados podrían servir de insumo y soporte para las organizaciones, puesto que la identificación y el entendimiento de los factores valorados por los consumidores y las relaciones entre ellos, es parte fundamental en la toma de decisiones organizacionales; con lo que se pueden realizar acciones que permitan mejorar la calidad de servicio percibida por los usuarios. Lo anterior, podría incidir en la reputación, aumento de usuarios, utilidades; entre otros beneficios. 


\section{REFERENCIAS}

Abascal, E., \& Grande, I. (2005). Análisis de encuestas. Madrid: ESIC Editorial.

Anderson, J. C., \& Gerbing, D. W. (1988). Structural Equation Modeling in Practice: A Review and Recommended Two-Step Approach. Psychological Bulletin, 103(3), 411.

Bagozzi, R. P., \& Yi, T. (1988). On the Evaluation of Structural Equation Models. Journal of the Academy of Marketing Science, 16(1), 74-94.

Bernal, A. (2014). Fidelización de clientes en organizaciones deportivas: calidad, valor percibido y satisfacción como factores determinantes (tesis doctoral). Recuperado de https://idus.us.es/xmlui/handle/11441/53492

Calabuig, F., Burillo, P., Crespo, J., Mundina, J. J., \& Gallardo, L. (2010). Satisfacción, calidad y valor percibido en espectadores de atletismo. Revista Internacional de Medicina y Ciencias de la Actividad Física y el Deporte, 10(40), 577-593. https://doi.org/Http://cdeporte.rediris.es/revista/revista40/artsatisfaccion182.htm

Calabuig, F., Quintanilla, I., \& Mundina, J. (2008). La calidad percibida de los servicios deportivos: diferencias según instalación, género, edad y tipo de usuario en servicios náuticos. Revista Internacional de Ciencias del Deporte, 3(IV), 25-43. https://doi.org/10.5232/ricyde2008.010.03

Calvo, C., Martínez, V. A., \& Juanatey, O. (2013). Análisis de dos modelos de ecuaciones estructurales alternativos para medir la intención de compra. Revista Investigación Operacional, 34(3), 230243.

Chhabra, N. (2013). Medición de la percepción del consumidor de la calidad del servicio en la venta al por menor organizada mediante el instrumento Servqual. Management Dynamics, 13(1), 7082.

Cramer, H. (1946). Mathematical Methods of Statistics. New Jersey: Princeton University Press. https://doi.org/doi:10.1111/ejss.12473

Cronin, J. J., \& Taylor, S. A. (1992). Measuring Service Quality: A Reexamination and Extension. Journal of Marketing, 56(3), 55-68. https://doi.org/10.2307/1252296

Cuadrado, J. (2016). ¿Acabaremos trabajando todos en servicios? Cuadernos de Información Económica, 250, 73-86.

Dhurup, M., Singh, P. C., \& Surujlal, J. (2006). Customer service quality at commercial health and fitness centres. South African Journal for Research in Sport, Physical Education and Recreation, 28(2), 39-54.

Duque, E. J., \& Pinzón-Chaparro, C. R. (2012). Medición de la percepción de la calidad del servicio de educación por parte de los estudiantes de la UPTC Duitama. Criterio Libre, 10(16), 159-192. 
Factores asociados a la calidad en el servicio en Centros de Acondicionamiento Físico a partir del modelo SERVQUAL

Fierro, J. (2010). Análisis estadístico univariado, bivariado y variables control. Recuperado de http://chitita.uta.cl/cursos/2012-1/0000104/recursos/r-25.pdf

Fragoso, J. T., \& Espinoza, I. L. (2017). Assessment of banking service quality perception using the SERVPERF model. Contaduría y Administración, 62(4), 1270-1293.

Gálvez, P., \& Morales, V. (2011). Evaluación de la calidad en programas municipales deportivos: generalizabilidad y optimización de diseños de medida. Cuadernos de Psicología del Deporte, 11(2), 123-130.

García, A. (2016). Service culture in customer service improvement. Telos, 18(3), 381-398.

García, J., Bernal, A., Lara, A., \& Galán, P. (2013). Influence of perceived service quality on loyalty among elderly users of public fitness centres. Escritos de Psicología, 6(2), 26-34. https://doi.org/10.5231/psy.writ.2013.2206

García, J., Cepeda, G., \& Martín, D. (2012). La satisfacción de clientes y su relación con la percepción de calidad en Centro de Fitness: utilización de la escala CALIDFIT. Revista de Psicología del Deporte, 21(2), 309-319.

George, S., \& Mallery, L. (2003). Alfa de Cronbach y consistencia interna de los ítems de un instrumento de medida. Revista de estudios Interdisciplinarios en Ciencias Sociales, 3(16), 3-9.

Goetsch, D. L., \& Davis, S. (2013). Quality Management for Organizational Excellence: Introduction to Total Quality (7th ed.). Boston: Pearson.

Grajales, T. (2000). Tipos de investigación. Investipos. Retrieved from http://tgrajales.net/investipos.pdf

Gulc, A. (2016). Models and Methods of Measuring the Quality of Logistic Service. In 7th International Conference on Engineering, Project, and Production Management (pp. 255-264). Bialystok, Poland: by Elsevier. https://doi.org/doi: 10.1016/j.proeng.2017.03.187

Hair, J. F., Anderson, R. E., Tatham, R. L., \& Black, W. C. (2001). Análisis Multivariante (5ta ed.). Madrid: Prentice-Hall.

Hemalatha, S., Dumpala, L., \& Balakrishna, B. (2018). Service quality evaluation and ranking of container terminal operators through hybrid multi-criteria decision making methods. Asian Journal of Shipping and Logistics, 34(2), 137-144.

Kaiser, H. F. (1970). A Second-generation Little Jiffy. Psychometrika, 25, 401-415. https://doi.org/10.1007/BF02291817

Manulik, S., Rosińczuk, J., \& Karniej, P. (2016). Evaluation of health care service quality in Poland with the use of SERVQUAL method at the specialist ambulatory health care center. Patient Preference and Adherence, 10(1), 1435-1442. https://doi.org/10.2147/PPA.S108252 
Martínez, J. A., \& Martínez, L. (2010). Some insights on conceptualizing and measuring service quality. Journal of Retailing and Consumer Services, 17(1), 29-42. https://doi.org/10.1016/j.jretconser.2009.09.002

Maul, A., Mari, L., Torres, D., \& Wilson, M. (2018). The quality of measurement results in terms of the structural features of the measurement process. Measurement, 116, 611-620.

Mavrou, I. (2015). Análisis factorial exploratorio: cuestiones conceptuales y metodológicas. Revista nebrija de lingüistica aplicada a la enseñanza de las lenguas, 1(19). Recuperado de http://www.nebrija.com/revista-linguistica/analisis-factorial-exploratorio

Midor, K., \& Kučera, M. (2018). Improving the Service with the Servqual Method. Management Systems in Production Engineering, 26(1), 60-65. https://doi.org/10.2478/mspe-2018-0010

Morales, V., Hernández-Mendo, A., \& Blanco, A. (2005). Evaluación de la calidad de los programas de actividad física. Psicothema, 17(2), 311-317.

Morquecho-Sánchez, R., Medina-Rodríguez, R., Morales-Sánchez, V., Medina, M., \& Duelos-Martínez, J. (2013). Enfoque diacrónico del cuestionario SERVQUAL para su aplicación a entidades deportivas. En I/I Congreso de la Asociación Latinoamericana de Gerencia Deportiva. Recuperado de http://eprints.uanl.mx/id/eprint/4424

Murillo, C., \& Saurina, C. (2013). Measurement of the importance of user satisfaction dimensions in healthcare provision. Gaceta Sanitaria, 27(4), 304-309. https://doi.org/10.1016/j.gaceta.2012.12.015

Ou, D., Mak, C. M., \& Pan, S. (2017). A method for assessing soundscape in urban parks based on the service quality measurement models. Applied Acoustics, 127, 184-193. https://doi.org/ 10.1016/j.apacoust.2017.06.006

Oviedo, H. C., \& Campo-Arias, A. (2005). An Approach to the Use of Cronbach's Alfa. Revista Colombiana de Psiquiatría, 34(4), 572-580.

Panigrahi, S. K., Azizan, N. A., \& Ali Khan, M. W. (2018). Investigating the Empirical Relationship Between Service Quality, Trust, Satisfaction, and Intention of Customers Purchasing Life Insurance Products. Indian Journal of Marketing, 48(1), 28-46. https://doi.org/10.1016/j.apacoust.2017.06.006

Parasuraman, A., Zeithaml, V., \& Berry, L. (1988). SERVQUAL: A Multiple-Item Scale for Measuring Consumer Perceptions of Service Quality. Journal of Retailing, 64(1), 12-40.

Ramírez, C., Lorenzo, A., \& López, C. (2017). Revisión de los instrumentos de medida de la calidad en los servicios deportivos. Journal Kronos, 16(1).

Rial, J., Varela, J., Rial, A., \& Ral, E. (2010). Modelización y medida de la calidad percibida en centros 
Factores asociados a la calidad en el servicio en Centros de Acondicionamiento Físico a partir del modelo SERVQUAL

deportivos: la escala QSport-10. Revista Internacional de Ciencias del Deporte, 18, 57-73. https://dx.doi.org/10.5232/ricyde2010.01804

Serrano, J. A., \& Segado, F. S. (2015). Análisis de las herramientas de medición de percepción de la calidad en los servicios deportivos: revisión de la literatura. Cultura, Ciencia y Deporte, 10(28), 67-76. http://dx.doi.org/10.12800/ccd.v10i28.516

Takenaka, T., Nishino, N., \& Nishikori, H. (2018). Service benchmarking for the co-creation of service ecosystem. Procedia CIRP, 67, 574-576. https://doi.org/10.1016/B978-0-08-098285-4.00004-2

Theodorakis, N., Kambitsis, C., \& Laios, A. (2001). Relationship between measures of service quality and satisfaction of spectators in professional sports. An International Journal, 11(6), 431-438. https://doi.org/10.1108/09604520110410638

Vargas, M., \& Aldana, L. (2015). Calidad y servicio conceptos y herramientas (3rd ed.). Bogotá, Colombia: ECOE Ediciones. 